\title{
On Three-dimensional Formation Control with Mismatched Coordinates
}

\author{
Ziyang Meng, Brian D. O. Anderson and Sandra Hirche
}

\begin{abstract}
A typical formation shape control problem involves point agents sensing relative positions, i.e. orientations and distances, of their neighbors and then moving so that these relative positions achieve some prespecified values. Such a procedure, requiring as it does sensing of orientations, implicitly presupposes that all agents have a shared understanding of the common orientations. On the other hand, there may be biases in sensors, variations in the earth's magnetic field interfering with compass-based sensing, or drift in inertial sensors, with the result that orientations are inconsistently measured or measured with error. In this paper, we investigate the formation control problem with mismatched coordinates in the threedimensional space, considering the consequences of this error. First, the situation of a two agent formation is first considered. We show that the agents converge to a fixed, but distorted formation exponentially fast. In contrast to the matched case, the formation is not asymptotically stationary, but rather instead translates with a certain constant velocity depending on the mismatches. The formation distortion between the actual one and the desired one is obtained, as well as the steady state velocity of the formation for small mismatch orientations. The case of agents with double integrator dynamics is then considered and similar phenomena are observed. Based on the results, an estimation algorithm is given to obtain the mismatch rotation matrix, which allows a compensation algorithm to be proposed such that the desired formation is achieved with zero steady-state velocity for the formation as a whole. The case of $n$-agent formations is finally considered, first with a star graph and then with a general graph. Simulations are provided to validate the theoretical results.
\end{abstract}

\section{INTRODUCTION}

Formation shape control problems are considered an important issue in the study of multi-agent systems and shape control has broad applications [1], [2]. There are different variations for the formation shape control problem. For example, problems with or without a leader were considered in [3], [4]; problems with undirected or directed communication topology were studied in [5], [6] and problems with velocity consensus and moving final formation were investigated

Z. Meng is with the State Key Laboratory of Precision Measurement Technology and Instruments and Department of Precision Instrument, Tsinghua University, Beijing 100084, China. B. D. O. Anderson is with The Australian National University and National ICT Australia, Canberra ACT 2600 Australia. S. Hirche is with the Chair of Information-Oriented Control, Technical University of Munich, D-80290 Munich, Germany. Emails: ziyangmeng@tsinghua.edu.cn, brian. andersonaanu. edu.au, hirche@tum.de. Corresponding author: Z. Meng. NICTA is funded by the Australian Government under the ICT Centre of Excellence Program. This work was partly supported by National Science Foundation of China under Grant 61503249 , Beijing Municipal Natural Science Foundation under Grant 4173075, National Key Research and Development Plan under Grant 2016YFB0500900/2, NICTA, the Australian Research Council under grant DP130103610 and DP160104500, the German Research Foundation (DFG) within the Priority Program SPP 1914 "Cyber-Physical Networking" and the Institute for Advanced Study of Technical University of Munich. in [7], [8]. Another major distinction rests with problems where there are both a prescribed shape and a prescribed orientation, and problems seeking simply to achieve a prescribed shape. A linear consensus-based algorithm can be used to solve a formation shape control problem with both a prescribed shape and a prescribed orientation [9], [10] while the gradient-based approach (which involves nonlinear control) can be used for shape control without an orientation objective [11], [12]. In this paper, the consensus-based approach is considered and we are interested in studying the consequence of postulating the existence of errors in relative state measurements. In particular, distance errors have been considered in the context of formation shape control without an orientation in [13], [14]. It was shown in [13] that if the agents have different understandings of either the desired distance between them, or of the actual distance between them, the resulting steady state formation will be of fixed shape but distorted relative to the desired shape (the amount of distortion depending on the mismatch). Further, instead of being stationary, the resulting formation shape will converge to a circular closed orbit in two-dimensional plane. The radius may be large, but the angular velocity is proportional to the mismatch. The extension to the case of a threedimensional tetrahedron formation shape control problem was considered in [14] and it was shown that the motion behavior is typically a helix and attributable to mismatch in desired or measured distances for a pair of agents.

We focus on a different mismatched quantity in this paper. It is evident that it will often be unrealistic to claim that all agents have common error-free knowledge of the orientations of north, or of the vertical direction. Biases can exist in instruments; drift can occur in inertial navigation systems; spatial variation can occur in the earth's magnetic field; horizon sensors may have difficulty sensing the horizon, and so on. We extend our previous work on this topic from a twodimensional case [15] to a three dimensional case. A brief version of this work has been published in [16]. A similar problem was considered in [17] with an orientation mismatch of local reference frames of the agents for the formation shape control problem. A combination algorithm aimed at both orientation alignment control and formation control was proposed. However, it is required that the orientation angles of all the local reference frames with respect to a global frame are available and can be exchanged among the neighbors. This somehow violates the spirit of distributed algorithms.

In this paper, we first consider the coordinate frame mismatch problem (in a three-dimensional ambient space) for the two agent case and then study the case with double 
integrator agents. In particular, we show that the agents converge to a fixed, but (relative to the desired one) distorted formation exponentially fast for both cases. The shape error between the actual final formation and the desired formation is properly defined and obtained. It is shown that the shape error magnitude is roughly proportional to the square of the angular mismatch in the coordinate axis orientations of the two agents. An estimation algorithm for the mismatched orientation is also proposed. Based on the design of the estimator, which is incorporated in a more complicated control, a mismatch compensation algorithm is proposed such that the desired formation is achieved and in steady state, it is stationary. We finally include discussions on the corresponding study of the $n$-agent case, including the issue of compensation.

Notation: $\|x\|$ denote the 2-norm of a vector $x \in \mathbb{R}^{d}$. Let $f$ and $g$ be two functions defined on some subset of the real numbers. One writes $f(x)=O(g(x))$ as $x \rightarrow 0$ if and only if there exist positive real numbers $M$ and $\delta$ such that $|f(x)| \leq M|g(x)|$ for $|x|<\delta$.

\section{PROBLEM FORMULATION}

To illustrate the key concepts, we start from the matched formation shape control for two agents in a three-dimensional space,

$$
\begin{aligned}
& \dot{\mathbf{A}}_{1}=\left(\mathbf{A}_{2}-\mathbf{A}_{1}\right)-\mathbf{D}, \\
& \dot{\mathbf{A}}_{2}=\left(\mathbf{A}_{1}-\mathbf{A}_{2}\right)+\mathbf{D},
\end{aligned}
$$

where $\mathbf{A}_{1}=\left[x_{1}, y_{1}, z_{1}\right]^{\mathrm{T}} \in \mathbb{R}^{3}$ and $\mathbf{A}_{2}=\left[x_{2}, y_{2}, z_{2}\right]^{\mathrm{T}} \in \mathbb{R}^{3}$ are the positions of agents 1 and $2, \mathbf{V}_{1}=\dot{\mathbf{A}}_{1}$ and $\mathbf{V}_{2}=$ $\dot{\mathbf{A}}_{2}$ represent the velocities of agents 1 and 2 , and $\mathbf{D}=$ $\left[d_{x}, d_{y}, d_{z}\right]^{\mathrm{T}} \in \mathbb{R}^{3}$ is a given desired relative position and known for each agent. The objective is to drive agents 1 and 2 to form a stationary formation such that $\mathbf{A}_{2}=\mathbf{A}_{1}+\mathbf{D}$. Note that the position of the centroid is unspecified, while the orientation and shape of the formation are specified. It is straightforward from (1) to show that $\lim _{t \rightarrow \infty}\left(\mathbf{A}_{2}(t)-\right.$ $\left.\mathbf{A}_{1}(t)\right)=\mathbf{D}, \lim _{t \rightarrow \infty} \dot{\mathbf{A}}_{1}(t)=0$, and $\lim _{t \rightarrow \infty} \dot{\mathbf{A}}_{2}(t)=0$ exponentially fast. Therefore, agents converge to the desired formation and the velocities converge to zero exponentially fast.

The above algorithm assumes that $\mathbf{A}_{2}-\mathbf{A}_{1}$ for agent 1 and $\mathbf{A}_{1}-\mathbf{A}_{2}$ for agent 2 are identical (up to the sign). This means that a global coordinate system is shared for the two agents. However, this assumption is unlikely to be satisfied in real systems. As already indicated, we will focus on the orientation (as opposed to range) error.

Without any loss of generality, we suppose that the global coordinates coincide with the coordinate basis of agent 1. We next derive the equation of motion of agent 2 in global coordinates. We also assume that the rotation from the coordinate frame of agent 1 to that of agent 1 is represented by an Euler axis $\mathbf{n}$ and an angle $\phi$ [18], where $\mathbf{n}=\left[\begin{array}{l}n_{1} \\ n_{2} \\ n_{3}\end{array}\right] \in \mathbb{R}^{3}$ is a unit vector. An illustration is given

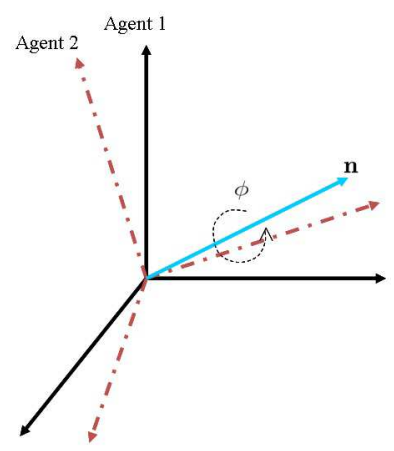

Fig. 1. Coordinates of agents 1 and 2 .

in Figure 1. Therefore, a line vector in global coordinates ${ }^{1} v=[x, y, z]^{\mathrm{T}}$ can be described in agent 2's coordinate basis as ${ }^{2} v=R(\mathbf{n}, \phi)^{1} v$, where $R(\mathbf{n}, \phi)$ is the rotation matrix [19], $R(\mathbf{n}, \phi)=I+\sin \phi[\mathbf{n}]_{\times}+(1-\cos \phi)\left([\mathbf{n}]_{\times}\right)^{2}$, and $[\mathbf{n}]_{\times}=\left[\begin{array}{ccc}0 & -n_{3} & n_{2} \\ n_{3} & 0 & -n_{1} \\ -n_{2} & n_{1} & 0\end{array}\right]$ denotes the cross product matrix of $\mathbf{n}$.

Then, in each agent's own coordinate basis, the actual kinematics of each agent with mismatched orientations are given by

$$
\begin{gathered}
{ }^{1} \dot{\mathbf{A}}_{1}=\mathbf{A}_{2}-\mathbf{A}_{1}-\mathbf{D}, \\
{ }^{2} \dot{\mathbf{A}}_{2}=R(\mathbf{n}, \phi)\left(\mathbf{A}_{1}-\mathbf{A}_{2}\right)+\mathbf{D},
\end{gathered}
$$

where $\mathbf{A}_{1}-\mathbf{A}_{2}$ is expressed in global coordinates, ${ }^{1} \dot{\mathbf{A}}_{1}$ and ${ }^{2} \dot{\mathbf{A}}_{2}$ are the velocity vectors of agents 1 and 2 expressed in each agent's own coordinate basis.

\section{Formations IN THREE-DIMENSIONAL SPACE}

It is not hard to show that (2) can be written as

$$
\begin{gathered}
\dot{\mathbf{A}}_{1}=\mathbf{A}_{2}-\mathbf{A}_{1}-\mathbf{D}, \\
\dot{\mathbf{A}}_{2}=\mathbf{A}_{1}-\mathbf{A}_{2}+R(\mathbf{n},-\phi) \mathbf{D},
\end{gathered}
$$

We next present the following result on the case of threedimensional ambient space.

Theorem 1: Consider the mismatched formation shape control algorithm (2). Suppose $\phi \neq 0$ is constant (the case of $\phi=0$ is just a classical formation control problem). It follows that

[Intra-formation motion]

(I) The agents converge to a fixed formation exponentially fast. In particular, $\lim _{t \rightarrow \infty}\left(\mathbf{A}_{2}(t)-\mathbf{A}_{1}(t)\right)=\frac{1}{2}(I+$ $R(\mathbf{n},-\phi)) \mathbf{D}$.

(II) The relative velocities of the agents converge to zero exponentially fast, i.e., $\lim _{t \rightarrow \infty}\left(\mathbf{V}_{2}(t)-\mathbf{V}_{1}(t)\right)=0$.

(III) If $\mathbf{D} \neq 0$, the final formation is distorted from the desired one. The formation distortion between the actual final one and the desired one is $O(|\phi|)$, as $\phi \rightarrow 0$. If $\mathbf{D}=0$, then rendezvous is still achieved, i.e., $\lim _{t \rightarrow \infty}\left(\mathbf{A}_{2}(t)-\mathbf{A}_{1}(t)\right)=0$. 
[Whole-formation motion]

(IV) If $\mathbf{D} \neq 0$, the absolute velocities of both agents converge to the same nonzero constant exponentially fast. In particular, $\lim _{t \rightarrow \infty}\left\|\mathbf{V}_{1}(t)\right\|=\lim _{t \rightarrow \infty}\left\|\mathbf{V}_{2}(t)\right\|=$ $O(|\phi|)$, as $\phi \rightarrow 0$. If $\mathbf{D}=0$, the absolute velocities of both agents converge to zero exponentially fast.

$(V)$ If $\mathbf{D} \neq 0$, the agent positions $\mathbf{A}_{1}(t)$ and $\mathbf{A}_{2}(t)$ are neither convergent nor bounded. If $\mathbf{D}=0$, $\lim _{t \rightarrow \infty} \mathbf{A}_{1}(t)=\lim _{t \rightarrow \infty} \mathbf{A}_{2}(t)=\frac{\mathbf{A}_{1}(0)+\mathbf{A}_{2}(0)}{2}$, where $\mathbf{A}_{1}(0)$ and $\mathbf{A}_{2}(0)$ are the initial states of agents 1 and 2 .

Proof: The proof of this Theorem (which is not especially difficult) is omitted due to space limitations. The reader is referred to [16] for more details.

Remark 1: The time-varying mismatches are not dealt with in any detail in this manuscript due to space limitations. The general conclusion is that the difference between the actual final formation and the desired one will converge to a bounded internal ultimately as long as time-varying mismatches are bounded.

Remark 2: For practical applications, a leader is often designated to determine the translation and orientation of the entire formation. For the case of leader-following formation control with mismatched coordinates, it turns out that the formation will become asymptotically stationary and the velocity error caused by angle measurement mismatch will be suppressed while the final formation is distorted from the desired one.

\section{SECOND-ORDER DYNAMICS}

Formation control problems have also been studied for agents consisting of double integrator dynamics [20], [21] since the dynamics of many motion systems are typically described by a second-order differential equation. We shall show that similar results to those for single integrator dynamics apply in this case.

With double integrator dynamics in each agent's own coordinate basis, the dynamics of each agent are given by

$$
\begin{gathered}
{ }^{1} \ddot{\mathbf{A}}_{1}=\mathbf{A}_{2}-\mathbf{A}_{1}-\mathbf{D}-{ }^{1} \dot{\mathbf{A}}_{1}, \\
{ }^{2} \ddot{\mathbf{A}}_{2}=R(\mathbf{n}, \phi)\left(\mathbf{A}_{1}-\mathbf{A}_{2}\right)+\mathbf{D}-{ }^{2} \dot{\mathbf{A}}_{2},
\end{gathered}
$$

where $\mathbf{A}_{1}-\mathbf{A}_{2},{ }^{1} \dot{\mathbf{A}}_{1}$ and ${ }^{2} \dot{\mathbf{A}}_{2}$ are the velocity vectors of agents 1 and 2 expressed in each agent's own coordinate basis, and ${ }^{1} \ddot{\mathbf{A}}_{1}$ and ${ }^{2} \ddot{\mathbf{A}}_{2}$ are the acceleration vectors of agents 1 and 2 expressed in each agent's own coordinate basis.

It is not hard to show that (4) can be written as

$$
\begin{gathered}
\ddot{\mathbf{A}}_{1}=\mathbf{A}_{2}-\mathbf{A}_{1}-\mathbf{D}-\dot{\mathbf{A}}_{1}, \\
\ddot{\mathbf{A}}_{2}=\mathbf{A}_{1}-\mathbf{A}_{2}+R(\mathbf{n},-\phi) \mathbf{D}-\dot{\mathbf{A}}_{2},
\end{gathered}
$$

We next present the following result on the case of secondorder dynamics.

Theorem 2: Consider the mismatched formation shape control algorithm (4). Suppose $\mathbf{n} \neq 0$ and $\phi \neq 0$ are constants. It follows that
[Intra-formation motion]

(I) The agents converge to a fixed formation exponentially fast. In particular, $\lim _{t \rightarrow \infty}\left(\mathbf{A}_{2}(t)-\mathbf{A}_{1}(t)\right)=\frac{1}{2}(I+$ $R(\mathbf{n},-\phi)) \mathbf{D}$.

(II) The relative velocities and accelerations of the agents converge to zero exponentially fast, i.e., $\lim _{t \rightarrow \infty}\left(\mathbf{V}_{2}(t)-\mathbf{V}_{1}(t)\right)=0$ and $\lim _{t \rightarrow \infty}\left(\ddot{\mathbf{A}}_{2}(t)-\right.$ $\left.\ddot{\mathbf{A}}_{1}(t)\right)=0$.

(III) If $\mathbf{D} \neq 0$, the final formation is distorted from the desired one. The formation distortion between the actual final one and the desired one is $O(|\phi|)$, as $\phi \rightarrow 0$. If $\mathbf{D}=0$, then rendezvous is still achieved, i.e., $\lim _{t \rightarrow \infty}\left(\mathbf{A}_{2}(t)-\mathbf{A}_{1}(t)\right)=0$.

[Whole-formation motion]

(IV) If $\mathbf{D} \neq 0$, the absolute velocities of both agents converge to the same nonzero constant exponentially fast. In particular, $\lim _{t \rightarrow \infty}\left\|\mathbf{V}_{1}(t)\right\|=\lim _{t \rightarrow \infty}\left\|\mathbf{V}_{2}(t)\right\|=$ $O(|\phi|)$, as $\phi \rightarrow 0$. If $\mathbf{D}=0$, the absolute velocities of both agents converge to zero exponentially fast.

(V) If $\mathbf{D} \neq 0$, the agent positions $\mathbf{A}_{1}(t)$ and $\mathbf{A}_{2}(t)$ are neither convergent nor bounded. If $\mathbf{D}=0$, $\lim _{t \rightarrow \infty} \mathbf{A}_{1}(t)=\lim _{t \rightarrow \infty} \mathbf{A}_{2}(t)=\frac{\mathbf{A}_{1}(0)+\mathbf{A}_{2}(0)}{2}$, where $\mathbf{A}_{1}(0)$ and $\mathbf{A}_{2}(0)$ are the initial states of agents 1 and 2.

Proof:

(I) Define $\mathbf{Q}=\mathbf{A}_{1}-\mathbf{A}_{2}+\frac{1}{2}(I+R(\mathbf{n},-\phi)) \mathbf{D}$. It follows from (5) that

$$
\ddot{\mathbf{Q}}=-2 \mathbf{Q}-\dot{\mathbf{Q}} \text {. }
$$

It then follows that $\lim _{t \rightarrow \infty}\left(\mathbf{A}_{2}(t)-\mathbf{A}_{1}(t)\right)=\overline{\mathbf{D}}$ exponentially, where $\overline{\mathbf{D}}=\frac{1}{2}(I+R(\mathbf{n},-\phi)) \mathbf{D}$. This verifies (I).

(II) Since $\lim _{t \rightarrow \infty}\left(\mathbf{A}_{2}(t)-\mathbf{A}_{1}(t)-\overline{\mathbf{D}}\right)=0$ exponentially fast, it follows from (6) that $\lim _{t \rightarrow \infty}\left(\dot{\mathbf{A}}_{2}(t)-\dot{\mathbf{A}}_{1}(t)\right)=0$ and $\lim _{t \rightarrow \infty}\left(\ddot{\mathbf{A}}_{2}(t)-\ddot{\mathbf{A}}_{1}(t)\right)=0$ exponentially fast. Therefore, (II) is proven.

(III) The proof of this part is the same as the proof of the corresponding part of Theorem 1.

(IV) It follows from (5) that

$$
\ddot{\mathbf{A}}_{1}=-\dot{\mathbf{A}}_{1}+\overline{\mathbf{D}}-\mathbf{D}+\mathbf{A}_{2}-\mathbf{A}_{1}-\overline{\mathbf{D}},
$$

it then follows from the input-to-state stability property ( [22]) that $\lim _{t \rightarrow \infty} \dot{\mathbf{A}}_{1}(t)=\overline{\mathbf{D}}-\mathbf{D}=\frac{1}{2}(R(\mathbf{n},-\phi)-I) \mathbf{D}=$ $\sin \frac{\phi}{2}\left(-\cos \frac{\phi}{2}[\mathbf{n}]_{\times}+\sin \frac{\phi}{2}\left([\mathbf{n}]_{\times}\right)^{2}\right) \mathbf{D}$ and $\lim _{t \rightarrow \infty} \ddot{\mathbf{A}}_{1}(t)=$ 0 . Therefore, we also have $\lim _{t \rightarrow \infty} \dot{\mathbf{A}}_{2}(t)=\frac{1}{2}(R(\mathbf{n},-\phi)-$ $I) \mathbf{D}$ and $\lim _{t \rightarrow \infty} \ddot{\mathbf{A}}_{2}(t)=0$. This further implies when $\mathbf{D} \neq 0$ that $\lim _{t \rightarrow \infty}\left\|\dot{\mathbf{A}}_{1}(t)\right\|=\lim _{t \rightarrow \infty}\left\|\dot{\mathbf{A}}_{2}(t)\right\|=O(|\phi|)$, as $\phi \rightarrow 0$. Therefore, for the case of $\mathbf{D} \neq 0$, the absolute velocities of both agents converge to the same nonzero constant exponentially fast. For the case of $\mathbf{D}=0$, the absolute velocities of both agents converge to zero exponentially fast.

(V) For the case of $\mathbf{D} \neq 0$, the conclusions are obvious due to (IV). If $\mathbf{D}=0$, the problem reduces to a standard average consensus problem [9].

Remark 3: Another interesting problem is to consider the case of relative velocity feedback with mismatched coordinates. In such a case, we conjecture that the absolute 


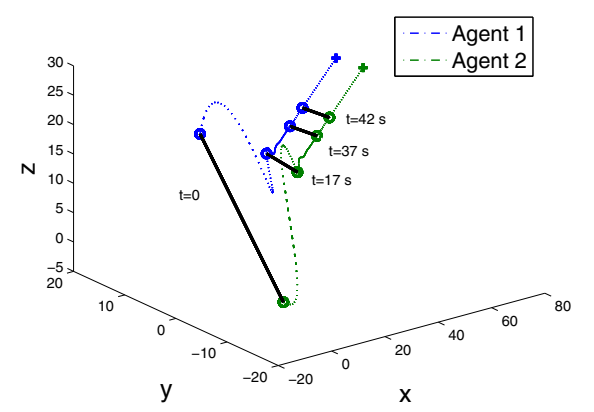

(a) The dotted lines denote the trajectories of the positions of the agents. The circles and the solid black lines denote respectively, the positions of the agents and the formation shape at $\mathrm{t}=17 \mathrm{~s}, \mathrm{t}=37 \mathrm{~s}$, and $\mathrm{t}=42 \mathrm{~s}$.

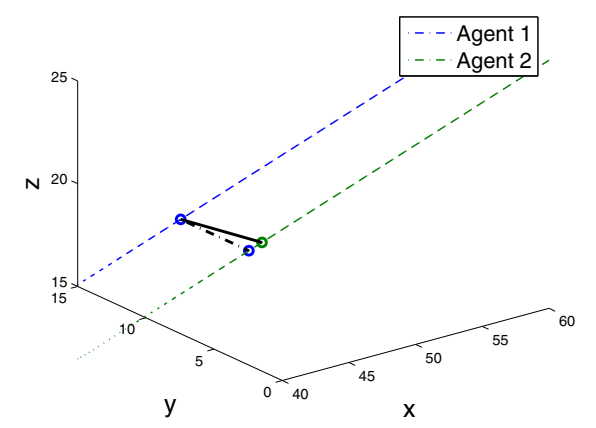

(b) The solid black line and the dotted black line denote respectively, the actual formation shape and desired formation shape at $\mathrm{t}=80 \mathrm{~s}$.

Fig. 2. Three agent case with $\mathbf{D} \neq 0$.

velocities of agents are not convergent and the accelerations of the agents converge to a common constant.

We next describe simulations which illustrate the validity of Theorem 2. We consider the case of $\mathbf{D} \neq 0$. In particular, $\mathbf{D}=[0,-5,0]^{\mathrm{T}}, \phi=-0.2 \pi$, and $\mathbf{n}_{1}=[1,0,0]^{\mathrm{T}}$ and initial states of all the agents are chosen randomly. Figures 2 and 3 show the positions and velocities of the agents during the time interval $[0,50]$. The relative velocities converge to zero, indicating that the agents converge to a fixed formation. However, due to the existence of mismatched sensors, the agents keep moving with non-zero constant absolute velocities and the final formation is distorted from the desired one.

\section{ESTIMATION AND COMPENSATION ALGORITHMS FOR TWO AGENT CASE}

In this section, we first consider the estimation of $R(\mathbf{n}, \phi)$. We shall show later how the use of an estimate of $R(\mathbf{n}, \phi)$ can eliminate the problem caused by the orientation mismatch. Without loss of generality, we let agent 2 be responsible for the estimation of $R(\mathbf{n}, \phi)$. The following compensation control algorithm is proposed

$$
\begin{aligned}
{ }^{1} \dot{\mathbf{A}}_{1} & =\mathbf{A}_{2}-\mathbf{A}_{1}-\mathbf{D}, \\
{ }^{2} \dot{\mathbf{A}}_{2} & =R(\mathbf{n}, \phi)\left(\mathbf{A}_{1}-\mathbf{A}_{2}\right)+\mathbf{D}+\mathbf{U},
\end{aligned}
$$

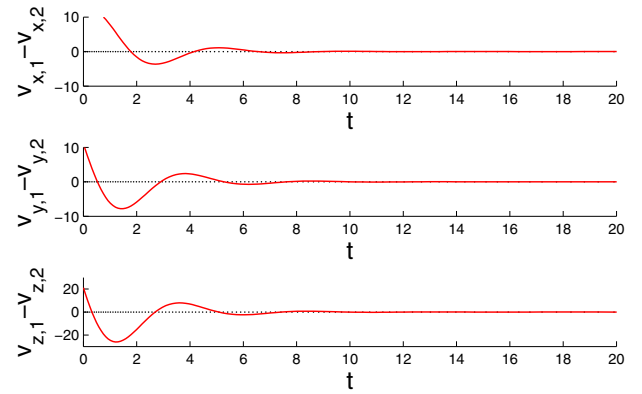

(a) The convergence of relative velocities of the agents.
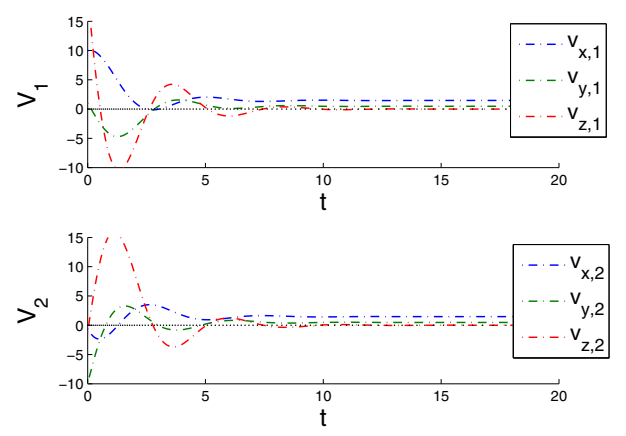

(b) The convergence of absolute velocities of the agents.

Fig. 3. Three agent case with $\mathbf{D} \neq 0$.

where $\mathbf{U} \in \mathbb{R}^{3}$ denotes a compensation input. We next specify how to design the estimation of $R(\mathbf{n}, \phi)$ and use the estimated information to compensate the original mismatched algorithm such that the desired formation is achieved.

\section{A. Estimation of $R(\mathbf{n}, \phi)$ using the TRIAD algorithm}

In this subsection, we use the measurement for agent 2 (relative position information expressed in the coordinate basis of agent 2 , i.e., ${ }^{2} \mathbf{A}_{2}-{ }^{2} \mathbf{A}_{1}$ ) and the measurement for agent 1 (relative position information expressed in the coordinate basis of agent 1 , i.e., ${ }^{1} \mathbf{A}_{2}-{ }^{1} \mathbf{A}_{1}$ ) for the TRIAD algorithm [23], [24] such that $R(\mathbf{n}, \phi)$ is reconstructed. The TRIAD algorithm is a classical deterministic algorithm to estimate an attitude, in which the attitude is directly calculated based on two pairs of nonparallel vectors. We next specify the TRIAD algorithm.

Suppose that we have two nonparallel unit vectors $V_{1}$ and $V_{2}$ (measurements of physical quantities obtained by agent 1 at two distinct time instants) and two other unit vectors $W_{1}$ and $W_{2}$ (measurements of the same physical quantities obtained by agent 2 at the same two distinct time instants). Clearly, the unknown rotation matrix $R(\mathbf{n}, \phi)$ defines the differences between them, where $W_{1}=R(\mathbf{n}, \phi) V_{1}$ and $W_{2}=R(\mathbf{n}, \phi) V_{2}$. Since $V_{1}$ and $V_{2}$ are linearly independent, we obviously have the following relation:

$$
\left(W_{1} \times W_{2}\right)=R(\mathbf{n}, \phi)\left(V_{1} \times V_{2}\right) .
$$

Motivated by the above fact, we can construct two triads of 
unit vectors:

$$
\begin{array}{ccc}
R_{1}=V_{1}, & R_{2}=\frac{V_{1} \times V_{2}}{\left\|V_{1} \times V_{2}\right\|}, & R_{3}=R_{1} \times R_{2}, \\
S_{1}=W_{1}, & S_{2}=\frac{W_{1} \times W_{2}}{\left\|W_{1} \times W_{2}\right\|}, & S_{3}=S_{1} \times S_{2},
\end{array}
$$

We thus know that

$$
M_{S}=R(\mathbf{n}, \phi) M_{R}
$$

where

$$
M_{S} \doteq\left[\begin{array}{lll}
S_{1} & S_{2} & S_{3}
\end{array}\right], \quad M_{R} \doteq\left[\begin{array}{lll}
R_{1} & R_{2} & R_{3}
\end{array}\right] .
$$

and the right members of the equations denote matrices labeled by their columns.

Lemma 1 (TRIAD algorithm): [23], [24] The matrices $M_{S}$ and $M_{R}$ are both orthogonal with determinant 1 and the solution for $R(\mathbf{n}, \phi)$ is

$$
R(\mathbf{n}, \phi)=M_{S} M_{R}^{-1}=M_{S} M_{R}^{\mathrm{T}} .
$$

\section{B. Compensation algorithm}

Next, we show how to use the estimate of $R(\mathbf{n}, \phi)$ to compensate the original mismatched algorithm such that the desired formation is achieved. Specifically, the following compensation control algorithm is proposed

$$
\mathbf{U}(t)=\left\{\begin{array}{l}
0, \quad t \in[0, T] \\
-\mathbf{D}+\hat{R} \mathbf{D}, \quad t \geq T
\end{array}\right.
$$

where $\hat{R}$ will be specified later.

We next show that $\mathbf{A}_{2}(t)-\mathbf{A}_{1}(t)$ converges to the desired formation using compensation input (9).

Theorem 3: Choose $V_{1}=\frac{\mathbf{A}_{2}(0)-\mathbf{A}_{1}(0)}{\left\|\mathbf{A}_{2}(0)-\mathbf{A}_{1}(0)\right\|}$ and $W_{1}=$ $\frac{R(\mathbf{n}, \phi)\left(\mathbf{A}_{2}(0)-\mathbf{A}_{1}(0)\right)}{\left\|R(\mathbf{n}, \phi)\left(\mathbf{A}_{2}(0)-\mathbf{A}_{1}(0)\right)\right\|}$. Also choose a $T>0$ such that $\left(\mathbf{A}_{2}(0)-\mathbf{A}_{1}(0)\right)^{\mathrm{T}}\left(\mathbf{A}_{2}(T)-\mathbf{A}_{1}(T)\right) \neq 0$. Then, choose $V_{2}=\frac{\mathbf{A}_{2}(T)-\mathbf{A}_{1}(T)}{\left\|\mathbf{A}_{2}(T)-\mathbf{A}_{1}(T)\right\|}$ and $W_{2}=\frac{R(\mathbf{n}, \phi)\left(\mathbf{A}_{2}(T)-\mathbf{A}_{1}(T)\right)}{\left\|R(\mathbf{n}, \phi)\left(\mathbf{A}_{2}(T)-\mathbf{A}_{1}(T)\right)\right\|}$ Consider the algorithm (8) with compensation input (9) and the estimator $\hat{R}=M_{S} M_{R}^{\mathrm{T}}$ derived according to the TRIAD algorithm. Then $\hat{R}=R(\mathbf{n}, \phi), \lim _{t \rightarrow \infty}\left(\mathbf{A}_{2}(t)-\mathbf{A}_{1}(t)\right)=$ $\mathbf{D}$, and $\lim _{t \rightarrow \infty} \mathbf{V}_{1}(t)=\lim _{t \rightarrow \infty} \mathbf{V}_{2}(t)=0$.

Proof: It is not hard to show from (8) and (9) $\mathbf{A}_{1}(t)-\mathbf{A}_{2}(t)$ is bounded during $t \in[0, T]$, and

$$
\dot{\mathbf{A}}_{1}-\dot{\mathbf{A}}_{2}=-2\left(\mathbf{A}_{1}-\mathbf{A}_{2}+\mathbf{D}\right)+\mathbf{D}-R^{\mathrm{T}}(\mathbf{n}, \phi) \hat{R} \mathbf{D}, \forall t \geq T .
$$

Therefore, according to Lemma 1, we know that

$$
\dot{\mathbf{A}}_{1}-\dot{\mathbf{A}}_{2}=-2\left(\mathbf{A}_{1}-\mathbf{A}_{2}+\mathbf{D}\right), \forall t \geq T .
$$

It then follows hat $\lim _{t \rightarrow \infty}\left(\mathbf{A}_{2}(t)-\mathbf{A}_{1}(t)\right)=\mathbf{D}$. It is therefore trivial to show that $\lim _{t \rightarrow \infty} \mathbf{V}_{1}(t)=\lim _{t \rightarrow \infty} \mathbf{V}_{2}(t)=$ 0 .

We next describe simulations which validate the effectiveness of Theorem 3. We consider $\mathbf{D}=[0,5,0]^{\mathrm{T}}$. The initial positions of the two agents are chosen randomly. From Figure 4, we can see that the absolute velocities of the agents converge to zero and the desired formation shape is achieved.

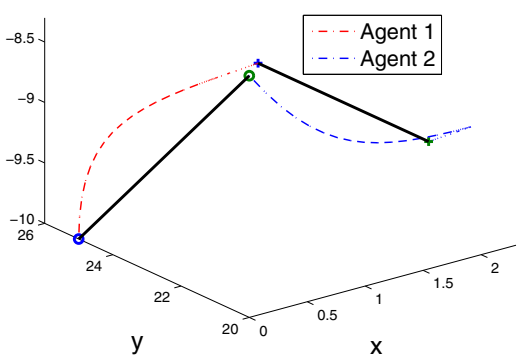

(a) The convergence of the positions of the agents. The circles and the crosses denote respectively, the initial and final positions of agents.
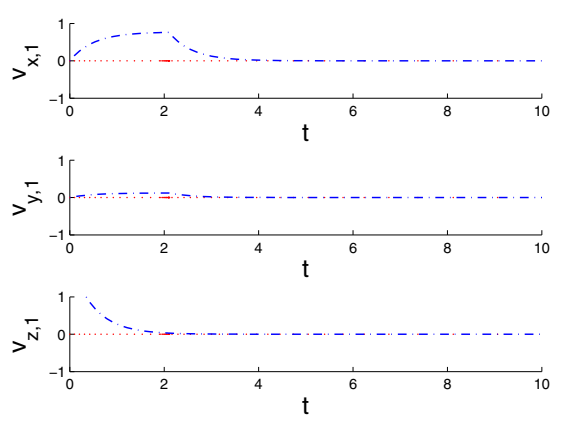

(b) The convergence of absolute velocities of the agents.

Fig. 4. Convergence of compensation algorithm (8) with compensation input (9).

Remark 4: We remark that the determination of $R(\mathbf{n}, \phi)$ is achieved with just two pairs of measurements. Obviously, if there is measurement noise, as opposed to bias or a systematic error of the type we are estimating, one would expect to do better with more measurements, or even continuous measurements. While the details are not developed in this paper, we note that extensions of the TRIAD algorithm to address such situations have been developed, see e.g., SVD method [25], FOAM method, [26], QUEST method [24], Euler-q method [27], and so on.

\section{THE $n$-AGENT CASE}

In this section, we aim to show how the analysis of the previous section for two agents will carry over to $n>$ 2 agents. For clarity of presentation, we first consider a special and simpler case of connected graphs, i.e., a star graph. Note that a star graph is a graph with minimum connectivity. Therefore, the case can be easily extended towards a general graph by expressing the desired and actual vectors corresponding to the edge filling in the network as a linear combination of those quantities for the star graph. We can therefore derive a general conclusion that holds for any connected graph of $n$ agents. 


\section{A. n-agent case with a star graph}

We consider that there are $n$ agents, $1,2, \ldots, n$ in a threedimensional space and agent $i$ is connected to agent 1 with an undirected edge, $\forall i=2,3, \ldots, n$. The classical formation control algorithm for each agent is given by:

$$
\begin{gathered}
\dot{\mathbf{A}}_{1}=\left(\mathbf{A}_{2}-\mathbf{A}_{1}\right)+ \\
+\mathbf{D}_{12}+\left(\mathbf{A}_{3}-\mathbf{A}_{1}\right)+\mathbf{D}_{13}+\ldots \\
+\left(\mathbf{A}_{n}-\mathbf{A}_{1}\right)+\mathbf{D}_{1 n} \\
\dot{\mathbf{A}}_{2}=\left(\mathbf{A}_{1}-\mathbf{A}_{2}\right)-\mathbf{D}_{12}
\end{gathered}
$$

$$
\dot{\mathbf{A}}_{n}=\left(\mathbf{A}_{1}-\mathbf{A}_{n}\right)-\mathbf{D}_{1 n},
$$

where $\mathbf{A}_{1}=\left[x_{1}, y_{1}\right]^{\mathrm{T}} \in \mathbb{R}^{2}, \mathbf{A}_{2}=\left[x_{2}, y_{2}\right]^{\mathrm{T}} \in \mathbb{R}^{2}, \ldots$, $\mathbf{A}_{n}=\left[x_{n}, y_{n}\right]^{\mathrm{T}} \in \mathbb{R}^{2}, \mathbf{D}_{12}=\left[d_{x, 12}, d_{y, 12}\right]^{\mathrm{T}} \in \mathbb{R}^{2}, \mathbf{D}_{13}=$ $\left[d_{x, 13}, d_{y, 13}\right]^{\mathrm{T}} \in \mathbb{R}^{2}, \ldots$ and $\mathbf{D}_{1 n}=\left[d_{x, 1 n}, d_{y, 1 n}\right]^{\mathrm{T}} \in \mathbb{R}^{2}$ are given constants and known for each agent.

The objective is to ensure asymptotically as $t \rightarrow \infty$ that $\mathbf{A}_{1}(t)-\mathbf{A}_{2}(t)=\mathbf{D}_{12}, \mathbf{A}_{1}(t)-\mathbf{A}_{3}(t)=\mathbf{D}_{13}, \ldots$, and $\mathbf{A}_{1}(t)-\mathbf{A}_{n}(t)=\mathbf{D}_{1 n}$. We define $\mathbf{D}=\left[\begin{array}{c}\mathbf{D}_{12} \\ \mathbf{D}_{13} \\ \vdots \\ \mathbf{D}_{1 n}\end{array}\right]$.

Due to the existence of mismatched compasses, and expressing measured multi-agent distances using the coordinate basis associated with agent 1 , the actual formation control algorithm becomes:

$$
\begin{gathered}
{ }^{1} \dot{\mathbf{A}}_{1}=\left(\mathbf{A}_{2}-\mathbf{A}_{1}\right)+\mathbf{D}_{12}+\left(\mathbf{A}_{3}-\mathbf{A}_{1}\right)+\mathbf{D}_{13}+\ldots \\
+\left(\mathbf{A}_{n}-\mathbf{A}_{1}\right)+\mathbf{D}_{1 n} \\
{ }^{2} \dot{\mathbf{A}}_{2}=R\left(\mathbf{n}_{2}, \phi_{2}\right)\left(\mathbf{A}_{1}-\mathbf{A}_{2}\right)-\mathbf{D}_{12}
\end{gathered}
$$

$$
{ }^{n} \dot{\mathbf{A}}_{n}=R\left(\mathbf{n}_{n}, \phi_{n}\right)\left(\mathbf{A}_{1}-\mathbf{A}_{n}\right)-\mathbf{D}_{1 n},
$$

where $\phi_{2}, \phi_{3}, \ldots, \phi_{n} \in(-\pi, \pi]$ denote the mismatch between the axes of agent 1 and agent $i, \forall i=2,3, \ldots, n$, $R\left(\mathbf{n}_{j}, \phi_{j}\right)=I+\sin \phi_{j}\left[\mathbf{n}_{j}\right]_{\times}+\left(1-\cos \phi_{j}\right)\left(\left[\mathbf{n}_{j}\right]_{\times}\right)^{2}$, where $\left[\mathbf{n}_{j}\right]_{\times}=\left[\begin{array}{ccc}0 & -n_{j, 3} & n_{j, 2} \\ n_{j, 3} & 0 & -n_{j, 1} \\ -n_{j, 2} & n_{j, 1} & 0\end{array}\right]$ denotes the cross product matrix of $\mathbf{n}_{j}$, for $\forall j=2,3, \ldots, n$.

It is not hard to show that (11) can be written as

$$
\begin{gathered}
\dot{\mathbf{A}}_{1}=\left(\mathbf{A}_{2}-\mathbf{A}_{1}\right)+\mathbf{D}_{12}+\left(\mathbf{A}_{3}-\mathbf{A}_{1}\right)+\mathbf{D}_{13}+\ldots \\
+\left(\mathbf{A}_{n}-\mathbf{A}_{1}\right)+\mathbf{D}_{1 n} \\
\dot{\mathbf{A}}_{2}=\left(\mathbf{A}_{1}-\mathbf{A}_{2}\right)-R\left(\mathbf{n}_{2},-\phi_{2}\right) \mathbf{D}_{12}
\end{gathered}
$$

$$
\dot{\mathbf{A}}_{n}=\left(\mathbf{A}_{1}-\mathbf{A}_{n}\right)-R\left(\mathbf{n}_{n},-\phi_{n}\right) \mathbf{D}_{1 n} .
$$

We next establish the following result.

Theorem 4: Consider the mismatched formation control algorithm (11). Suppose that $\phi_{2}, \phi_{3}, \ldots, \phi_{n} \neq 0$. It follows that

[Intra-formation motion]

(I) The agents converge to a fixed formation exponentially fast.

(II) The relative velocity of each agent pair converges to zero exponentially fast, i.e., $\lim _{t \rightarrow \infty}\left(\mathbf{V}_{i}(t)-\mathbf{V}_{j}(t)\right)=$ 0 , for all $i, j \in\{1,2, \ldots, n\}$.

(III) If $\mathbf{D} \neq 0$, the final formation is distorted from the desired one and the final formation distortion is $O\left(\max _{j=2,3, \ldots, n}\left|\phi_{j}\right|\right)$, as $\phi_{2}, \phi_{3}, \ldots, \phi_{n} \rightarrow 0$. If $\mathbf{D}=0, \lim _{t \rightarrow \infty}\left(\mathbf{A}_{i}(t)-\mathbf{A}_{j}(t)\right)=0$, for all $i, j \in$ $\{1,2, \ldots, n\}$.

[Whole-formation motion]

(IV) If $\mathbf{D} \neq 0$, the absolute velocity of each agent converges to the same nonzero constant exponentially fast. In particular, $\lim _{t \rightarrow \infty}\left\|\mathbf{V}_{i}(t)\right\|=O\left(\max _{j=2,3, \ldots, n}\left|\phi_{j}\right|\right)$, as $\phi_{2}, \phi_{3}, \ldots, \phi_{n} \rightarrow 0$, for all $i \in\{1,2, \ldots, n\}$.

(V) If $\mathbf{D} \neq 0$, the agent positions $\mathbf{A}_{1}(t), \mathbf{A}_{2}(t)$ and $\mathbf{A}_{3}(t)$ are neither convergent nor bounded. If $\mathbf{D}=$ $0, \lim _{t \rightarrow \infty} \mathbf{A}_{1}(t)=\cdots=\lim _{t \rightarrow \infty} \mathbf{A}_{n}(t)=$ $\frac{\mathbf{A}_{1}(0)+\cdots+\mathbf{A}_{n}(0)}{n}$, where $\mathbf{A}_{1}(0), \mathbf{A}_{2}(0), \ldots, \mathbf{A}_{n}(0)$ are the initial states of agents $1,2, \ldots, n$.

Proof:

(I) The intra-formation motion is determined by the following equation:

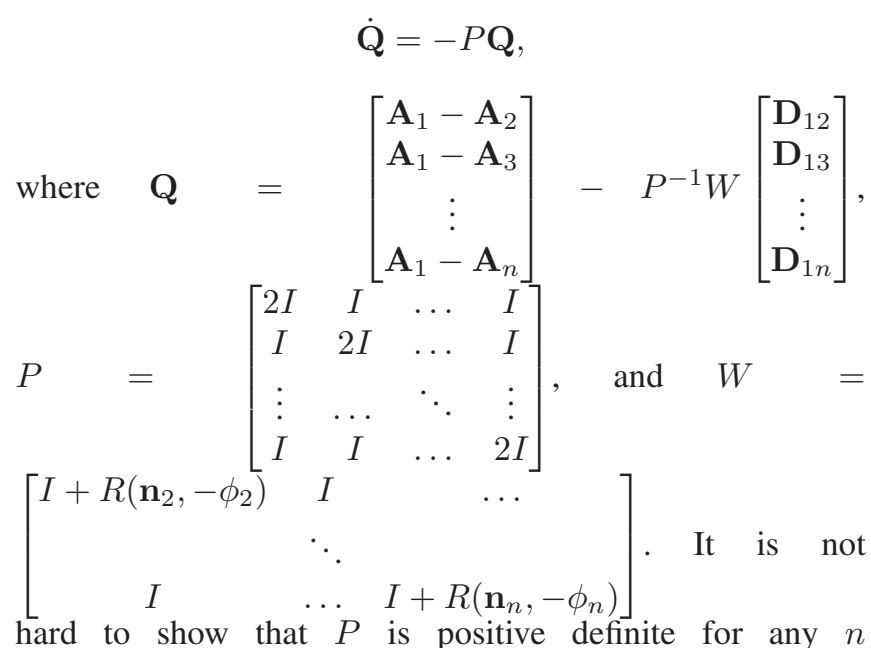
hard to show that $P$ is positive definite for any $n$ $\lim _{t \rightarrow \infty} \mathbf{Q}(t)=0$.

(II) It follows from (I) that $\lim _{t \rightarrow \infty}\left[\begin{array}{c}\dot{\mathbf{A}}_{1}(t)-\dot{\mathbf{A}}_{2}(t) \\ \dot{\mathbf{A}}_{1}(t)-\dot{\mathbf{A}}_{3}(t) \\ \vdots \\ \dot{\mathbf{A}}_{1}(t)-\dot{\mathbf{A}}_{n}(t)\end{array}\right]=0$. 
(III) For the case of $\mathbf{D} \neq 0$, we know that the actual final formation is determined by

$$
\lim _{t \rightarrow \infty}\left[\begin{array}{c}
\mathbf{A}_{1}(t)-\mathbf{A}_{2}(t) \\
\mathbf{A}_{1}(t)-\mathbf{A}_{3}(t) \\
\vdots \\
\mathbf{A}_{1}(t)-\mathbf{A}_{n}(t)
\end{array}\right]=P^{-1} W\left[\begin{array}{c}
\mathbf{D}_{12} \\
\mathbf{D}_{13} \\
\vdots \\
\mathbf{D}_{1 n}
\end{array}\right] .
$$

However, the desired formation is determined by $\left[\begin{array}{c}\mathbf{D}_{12} \\ \mathbf{D}_{13} \\ \vdots \\ \mathbf{D}_{1 n}\end{array}\right]$. The final formation distortion is defined and given by

$$
\begin{aligned}
\delta \mathbf{D}:=\left\|P^{-1} W\left[\begin{array}{c}
\mathbf{D}_{12} \\
\mathbf{D}_{13} \\
\vdots \\
\mathbf{D}_{1 n}
\end{array}\right]-\left[\begin{array}{c}
\mathbf{D}_{12} \\
\mathbf{D}_{13} \\
\vdots \\
\mathbf{D}_{1 n}
\end{array}\right]\right\| \\
=\left\|P^{-1}\left[\begin{array}{ccc}
R\left(\mathbf{n}_{2},-\phi_{2}\right)-I & \ldots & 0 \\
0 & \ddots & \\
& \ldots & R\left(\mathbf{n}_{n},-\phi_{n}\right)-I
\end{array}\right]\left[\begin{array}{c}
\mathbf{D}_{12} \\
\mathbf{D}_{13} \\
\vdots \\
\mathbf{D}_{1 n}
\end{array}\right]\right\| \\
\leq l_{1} \max _{j=2,3, \ldots, n}\left|\phi_{j}\right|\|\mathbf{D}\|,
\end{aligned}
$$

where we have used the fact that $R\left(\mathbf{n}_{j},-\phi_{j}\right)-\mathbf{I}=$ $-2 \sin \frac{\phi_{j}}{2}\left(\cos \frac{\phi_{j}}{2}\left[\mathbf{n}_{j}\right]_{\times}-\sin \frac{\phi_{j}}{2}\left(\left[\mathbf{n}_{j}\right]_{\times}\right)^{2}\right), \forall j=2,3, \ldots, n$, and $l_{1}$ is a constant. Therefore, $\delta \mathbf{D}=O\left(\max _{j=2,3, \ldots, n}\left|\phi_{j}\right|\right)$, as $\phi_{2}, \phi_{3}, \ldots, \phi_{n} \rightarrow 0$. In addition, it is trivial to prove the case of $\mathbf{D}=0$.

(IV) For the case of $\mathbf{D} \neq 0$, it follows from (11) that

$$
\begin{aligned}
& \lim _{t \rightarrow \infty} \dot{\mathbf{A}}_{1}(t)=\left[\begin{array}{llll}
-I & -I & \ldots & -I
\end{array}\right]\left[\begin{array}{c}
\mathbf{A}_{1}(t)-\mathbf{A}_{2}(t) \\
\mathbf{A}_{1}(t)-\mathbf{A}_{3}(t) \\
\vdots \\
\mathbf{A}_{1}(t)-\mathbf{A}_{n}(t)
\end{array}\right] \\
& +\left[\begin{array}{llll}
I & I & \ldots & I
\end{array}\right]\left[\begin{array}{c}
\mathbf{D}_{12} \\
\mathbf{D}_{13} \\
\vdots \\
\mathbf{D}_{1 n}
\end{array}\right]
\end{aligned}
$$$$
=\left[\begin{array}{llll}
-I & -I & \ldots & -I
\end{array}\right] P^{-1}(W-P)\left[\begin{array}{c}
\mathbf{D}_{12} \\
\mathbf{D}_{13} \\
\vdots \\
\mathbf{D}_{1 n}
\end{array}\right]
$$$$
=\left[\begin{array}{lll}
-\frac{1}{n} R\left(\mathbf{n}_{2},-\phi_{2}\right)+\frac{1}{n} I & \ldots & -\frac{1}{n} R\left(\mathbf{n}_{n},-\phi_{n}\right)+\frac{1}{n} I
\end{array}\right] \mathbf{D} .
$$

Therefore, the absolute velocities obey $\lim _{t \rightarrow \infty}\left\|\dot{\mathbf{A}}_{1}(t)\right\|=\cdots=\lim _{t \rightarrow \infty}\left\|\dot{\mathbf{A}}_{n}(t)\right\|=$ $O\left(\max _{j=2,3, \ldots, n}\left|\phi_{j}\right|\right)$, as $\phi_{2}, \phi_{3}, \ldots, \phi_{n} \rightarrow 0$. In addition, it is trivial to prove the claim for the case of $\mathbf{D}=0$.

Based on (IV), (V) is obvious.

B. Estimation and compensation algorithms in the n-agent case with star graph

Obviously it is of interest to be able to eliminate unwanted motions in the $n$-agent case, and this can be in fact be done by using effectively the same algorithm as proposed in the two agent case. Through measurements between communicating agent pairs $A_{1}$ and $A_{j}, j=2,3, \ldots, n$, the necessary compensation matrices are straightforwardly determined and the necessary compensation introduced.

\section{The n-agent case with a general graph}

Notation: an undirected graph $\mathcal{G}$ consists of a pair $(\mathcal{V}, \mathcal{E})$, where $\mathcal{V}=\{1,2, \ldots, n\}$ is a finite, nonempty set of nodes and $\mathcal{E} \subseteq \mathcal{V} \times \mathcal{V}$ is a set of unordered pairs of nodes. An edge $\{j, i\} \in \mathcal{E}$ denotes that nodes $i, j$ can obtain each other's information mutually. The neighbors of node $i$ are denoted by $\mathcal{N}_{i}:=\{j:\{j, i\} \in \mathcal{E}\}$. The adjacency matrix $\bar{A}=$ $\left[a_{i j}\right] \in \mathbb{R}^{n \times n}$ associated with the graph $\mathcal{G}$ is defined such that $a_{i j}=1$ if $\{j, i\} \in \mathcal{E}$ and $a_{i j}=0$ otherwise. It is obvious that $a_{i j}=a_{j i}$, for all $i, j \in \mathcal{V}$ for the undirected graph.

The classical formation control algorithm for each agent is given by:

$$
\dot{\mathbf{A}}_{i}=\sum_{j \in \mathcal{N}_{i}}\left(\mathbf{A}_{j}-\mathbf{A}_{i}+\mathbf{D}_{i j}\right), i \in \mathcal{V}
$$

where $\mathbf{A}_{i}=\left[x_{i}, y_{i}\right]^{\mathrm{T}} \in \mathbb{R}^{2}, \forall i \in \mathcal{V}, \mathbf{D}_{i j}=\left[d_{x, i j}, d_{y, i j}\right]^{\mathrm{T}} \in$ $\mathbb{R}^{2}, \forall i, j \in \mathcal{V}$ are given constants defining the specified relative positions and known for each agent, $\mathcal{N}_{i}, i \in \mathcal{V}$ denotes the neighbor set of agent $i$.

Due to the existence of mismatched compasses, and expressing measured multi-agent distances using the coordinate basis associated with agent 1 (without loss of generality), the actual formation control algorithm becomes:

$$
\begin{aligned}
{ }^{1} \dot{\mathbf{A}}_{1} & =\sum_{j \in \mathcal{N}_{1}}\left(\mathbf{A}_{j}-\mathbf{A}_{1}+\mathbf{D}_{1 j}\right), \\
{ }^{2} \dot{\mathbf{A}}_{2}= & \sum_{j \in \mathcal{N}_{2}}\left(R\left(\mathbf{n}_{2}, \phi_{2}\right)\left(\mathbf{A}_{j}-\mathbf{A}_{2}\right)+\mathbf{D}_{2 j}\right), \\
& \vdots \\
{ }^{n} \dot{\mathbf{A}}_{n}= & \sum_{j \in \mathcal{N}_{n}}\left(R\left(\mathbf{n}_{n}, \phi_{n}\right)\left(\mathbf{A}_{j}-\mathbf{A}_{n}\right)+\mathbf{D}_{n j}\right),
\end{aligned}
$$

where $\phi_{2}, \phi_{3}, \ldots, \phi_{n} \in(-\pi, \pi]$ denote the angular mismatch between the coordinate axes of agent 1 and agent $i, \forall i=2,3, \ldots, n, R\left(\mathbf{n}_{j}, \phi_{j}\right)=I+\sin \phi_{j}\left[\mathbf{n}_{j}\right]_{\times}+(1-$ $\left.\cos \phi_{j}\right)\left(\left[\mathbf{n}_{j}\right]_{\times}\right)^{2}$, where $\left[\mathbf{n}_{j}\right]_{\times}=\left[\begin{array}{ccc}0 & -n_{j, 3} & n_{j, 2} \\ n_{j, 3} & 0 & -n_{j, 1} \\ -n_{j, 2} & n_{j, 1} & 0\end{array}\right]$ denotes the cross product matrix of $\mathbf{n}_{j}$, for $\forall j=2,3, \ldots, n$.

It is not hard to show that (14) can be written as

$$
\begin{aligned}
\dot{\mathbf{A}}_{1} & =\sum_{j \in \mathcal{N}_{1}}\left(\mathbf{A}_{j}-\mathbf{A}_{1}+\mathbf{D}_{1 j}\right), \\
\dot{\mathbf{A}}_{2}= & \sum_{j \in \mathcal{N}_{2}}\left(\mathbf{A}_{j}-\mathbf{A}_{2}+R\left(\mathbf{n}_{2},-\phi_{2}\right) \mathbf{D}_{2 j}\right), \\
& \vdots \\
\dot{\mathbf{A}}_{n}= & \sum_{j \in \mathcal{N}_{n}}\left(\mathbf{A}_{j}-\mathbf{A}_{n}+R\left(\mathbf{n}_{n},-\phi_{n}\right) \mathbf{D}_{n j}\right) .
\end{aligned}
$$


We next establish the following result for the case of $n$ agents with a general connected graph.

Theorem 5: Consider the mismatched formation control algorithm (14). Suppose that $\phi_{2}, \phi_{3}, \ldots, \phi_{n} \neq 0$ with all $\phi$ constant for all time. It follows that

[Intra-formation motion]

(I) The agents converge to a fixed formation exponentially fast.

(II) The relative velocity of each agent pair converges to zero exponentially fast, i.e., $\lim _{t \rightarrow \infty}\left(\mathbf{V}_{i}(t)-\mathbf{V}_{j}(t)\right)=$ 0 , for all $i, j \in \mathcal{V}$.

(III) If $\mathbf{D} \neq 0$, the final formation is distorted from the desired one and the final formation distortion is $O\left(\max _{j=2,3, \ldots, n}\left|\phi_{j}\right|\right)$, as $\phi_{2}, \phi_{3}, \ldots, \phi_{n} \rightarrow 0$. If $\mathbf{D}=$ $0, \lim _{t \rightarrow \infty}\left(\mathbf{A}_{i}(t)-\mathbf{A}_{j}(t)\right)=0$, for all $i, j \in \mathcal{V}$.

[Whole-formation motion]

(IV) If $\mathbf{D} \neq 0$, the absolute velocity of each agent converges to the same nonzero constant exponentially fast. In particular, $\lim _{t \rightarrow \infty}\left\|\mathbf{V}_{i}(t)\right\|=O\left(\max _{j=2,3, \ldots, n}\left|\phi_{j}\right|\right)$, as $\phi_{2}, \phi_{3}, \ldots, \phi_{n} \rightarrow 0$, for all $i \in \mathcal{V}$.

(V) If $\mathbf{D} \neq 0$, the agent position $\mathbf{A}_{i}(t), \forall i \in \mathcal{V}$ is neither convergent nor bounded. If $\mathbf{D}=0, \lim _{t \rightarrow \infty} \mathbf{A}_{1}(t)=$ $\cdots=\lim _{t \rightarrow \infty} \mathbf{A}_{n}(t)=\frac{\mathbf{A}_{1}(0)+\cdots+\mathbf{A}_{n}(0)}{n}$, where $\mathbf{A}_{1}(0), \mathbf{A}_{2}(0), \ldots, \mathbf{A}_{n}(0)$ are the initial states of agents $1,2, \ldots, n$.

Proof: Using the relations that $\mathbf{A}_{j}-\mathbf{A}_{k}=\left(\mathbf{A}_{j}-\mathbf{A}_{1}\right)+$ $\left(\mathbf{A}_{1}-\mathbf{A}_{k}\right)$ and $\mathbf{D}_{k j}=\mathbf{D}_{1 j}-\mathbf{D}_{1 k}, \forall j, k \in \mathcal{V},(15)$ can be written as

$$
\begin{aligned}
\dot{\mathbf{A}}_{1}= & \sum_{j \in \mathcal{N}_{1}}\left(\mathbf{A}_{j}-\mathbf{A}_{1}+\mathbf{D}_{1 j}\right), \\
\dot{\mathbf{A}}_{2}= & N_{2}\left(\mathbf{A}_{1}-\mathbf{A}_{2}\right)-N_{2} R\left(\mathbf{n}_{2},-\phi_{2}\right) \mathbf{D}_{12} \\
& +\sum_{j \in \mathcal{N}_{2}}\left(\mathbf{A}_{j}-\mathbf{A}_{1}+R\left(\mathbf{n}_{2},-\phi_{2}\right) \mathbf{D}_{1 j}\right), \\
\vdots & N_{n}\left(\mathbf{A}_{1}-\mathbf{A}_{n}\right)-N_{n} R\left(\mathbf{n}_{n},-\phi_{n}\right) \mathbf{D}_{1 n} \\
& +\sum_{j \in \mathcal{N}_{n}}\left(\mathbf{A}_{j}-\mathbf{A}_{1}+R\left(\mathbf{n}_{n},-\phi_{n}\right) \mathbf{D}_{1 j}\right),
\end{aligned}
$$

where $N_{i}$ denotes the cardinality of set $\mathcal{N}_{i}, i \in \mathcal{V}$.

Therefore, the intra-formation motion can be

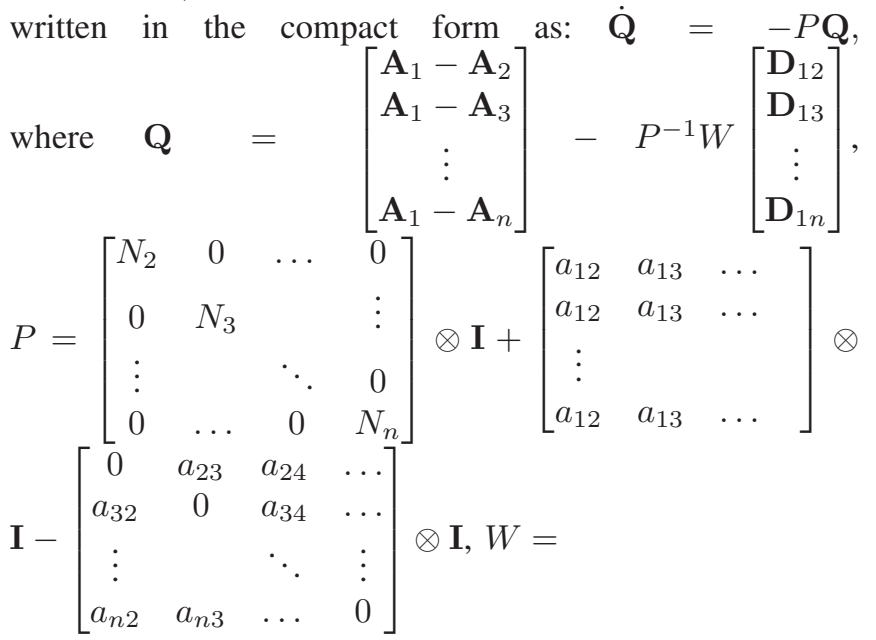

$$
\begin{aligned}
& {\left[\begin{array}{cccc}
N_{2} R\left(\mathbf{n}_{2},-\phi_{2}\right) & 0 & \cdots & 0 \\
0 & N_{3} R\left(\mathbf{n}_{3},-\phi_{3}\right) & & \vdots \\
\vdots & & \ddots & 0 \\
0 & \ldots & 0 & N_{n} R\left(\mathbf{n}_{n},-\phi_{n}\right)
\end{array}\right]+}
\end{aligned}
$$

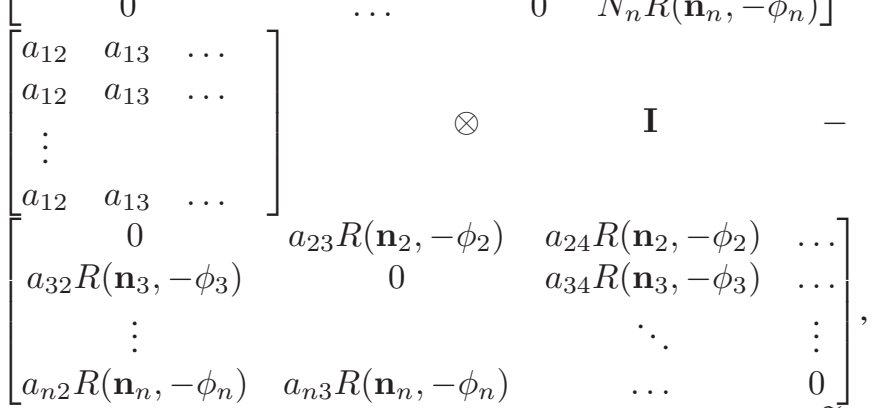

and $a_{i j}$ denotes $(i, j)$ th entry of the adjacency matrix $\widetilde{A}$. Based on the transformation and Lemma 1 given in ( [28]), it is not hard to show that $-P$ is a Hurwitz stable matrix when the underlying graph is connected. Therefore, $\lim _{t \rightarrow \infty} \mathbf{Q}(t)=0$.

(II) It follows from (I) that $\lim _{t \rightarrow \infty}\left(\dot{\mathbf{A}}_{1}(t)-\dot{\mathbf{A}}_{j}(t)\right)=0$, for all $j=2,3, \ldots, n$.

(III) For the case of $\mathbf{D} \neq 0$, we know that the actual final formation is determined by $\lim _{t \rightarrow \infty}\left[\begin{array}{c}\mathbf{A}_{1}(t)-\mathbf{A}_{2}(t) \\ \mathbf{A}_{1}(t)-\mathbf{A}_{3}(t) \\ \vdots \\ \mathbf{A}_{1}(t)-\mathbf{A}_{n}(t)\end{array}\right]=$ $P^{-1} W \mathbf{D}$. However, the desired formation is determined by D. The final formation distortion is defined and given by $\delta \mathbf{D}:=\left\|P^{-1} W \mathbf{D}-\mathbf{D}\right\|$. It then follows that

$$
\begin{aligned}
\delta \mathbf{D} & =\left\|P^{-1} \Omega \mathbf{D}\right\| \leq c_{1}\left\|\Omega_{1}\right\|\|\mathbf{D}\| \\
\leq & c_{1} \times \sqrt{2(n-1)} \times 2 \times \max _{j=2,3, \ldots, n}\left\{\left(N_{j}+\sum_{k=2}^{n} a_{j k}\right)\right. \\
& \left.\left|\sin \frac{\phi_{j}}{2}\right| \times 2\right\}\|\mathbf{D}\| \\
\leq & 4 c_{1} n \sqrt{2(n-1)}\|\mathbf{D}\| \max _{j=2,3, \ldots, n}\left|\phi_{j}\right|,
\end{aligned}
$$

where $\Omega$ and $\Omega_{1}$ are given in (22) and (23), respectively, $\Omega_{1, n n}=-N_{n} \sin \frac{\phi_{n}}{2}\left(\cos \frac{\phi_{n}}{2}\left[\mathbf{n}_{n}\right]_{\times}-\sin \frac{\phi_{n}}{2}\left(\left[\mathbf{n}_{n}\right]_{\times}\right)^{2}\right)$, $c_{1}=\left\|P^{-1}\right\|$ is a positive constant and we have used the facts that $R\left(\mathbf{n}_{j},-\phi_{j}\right)-\mathbf{I}=-2 \sin \frac{\phi_{j}}{2}\left(\cos \frac{\phi_{j}}{2}\left[\mathbf{n}_{j}\right]_{\times}-\right.$ $\left.\sin \frac{\phi_{j}}{2}\left(\left[\mathbf{n}_{j}\right]_{\times}\right)^{2}\right), j \in\{2,3, \ldots, n\}$ for the first inequality, $\|A\|_{2} \leq \sqrt{m}\|A\|_{\infty}=\sqrt{m} \max _{1 \leq i \leq m} \sum_{j=1}^{l}\left|a_{i j}\right|$ for a matrix $\mathbb{R}^{m \times l},|\sin \alpha| \leq 1$ and $|\cos \alpha| \leq 1, \forall \alpha \in \mathbb{R}$ for the second inequality. Therefore, $\delta \mathbf{D}=O\left(\max _{j=2,3, \ldots, n}\left|\phi_{j}\right|\right)$, as $\phi_{2}, \phi_{3}, \ldots, \phi_{n} \rightarrow 0$. In addition, it is trivial to prove the case of $\mathbf{D}=0$.

(IV) For the case of $\mathbf{D} \neq 0$, it follows from (15) that

$$
\begin{aligned}
& \dot{\mathbf{A}}_{1}(t)=\sum_{j \in \mathcal{N}_{1}}\left(\mathbf{A}_{j}-\mathbf{A}_{1}+\mathbf{D}_{1 j}\right)=\left[\begin{array}{llll}
-a_{12} & -a_{13} & \ldots & -a_{1 n}
\end{array}\right] \\
& \times\left[\begin{array}{c}
\mathbf{A}_{1}(t)-\mathbf{A}_{2}(t) \\
\mathbf{A}_{1}(t)-\mathbf{A}_{3}(t) \\
\vdots \\
\mathbf{A}_{1}(t)-\mathbf{A}_{n}(t)
\end{array}\right]+\left[\begin{array}{llll}
a_{12} & a_{13} & \ldots & a_{1 n}
\end{array}\right] \mathbf{D} .
\end{aligned}
$$




$$
\begin{gathered}
\Omega=\left[\begin{array}{cccc}
N_{2}\left(R\left(\mathbf{n}_{2},-\phi_{2}\right)-\mathbf{I}\right) & a_{23}\left(\mathbf{I}-R\left(\mathbf{n}_{2},-\phi_{2}\right)\right) & a_{24}\left(\mathbf{I}-R\left(\mathbf{n}_{2},-\phi_{2}\right)\right) & \ldots \\
a_{32}\left(\mathbf{I}-R\left(\mathbf{n}_{3},-\phi_{3}\right)\right) & N_{3}\left(R\left(\mathbf{n}_{3},-\phi_{3}\right)-\mathbf{I}\right) & a_{34}\left(\mathbf{I}-R\left(\mathbf{n}_{3},-\phi_{3}\right)\right) & \ldots \\
\vdots & \ddots & N_{n}\left(R\left(\mathbf{n}_{n},-\phi_{n}\right)-\mathbf{I}\right)
\end{array}\right] \\
\Omega_{1}=2\left[\begin{array}{cccc}
-N_{2} \sin \frac{\phi_{2}}{2}\left(\cos \frac{\phi_{2}}{2}\left[\mathbf{n}_{2}\right]_{\times}-\sin \frac{\phi_{2}}{2}\left(\left[\mathbf{n}_{2}\right]_{\times}\right)^{2}\right) & a_{23} \sin \frac{\phi_{2}}{2}\left(\cos \frac{\phi_{2}}{2}\left[\mathbf{n}_{2}\right]_{\times}-\sin \frac{\phi_{2}}{2}\left(\left[\mathbf{n}_{2}\right]_{\times}\right)^{2}\right) & \ldots & \ldots \\
a_{n 2}\left(\mathbf{I}-R\left(\mathbf{n}_{n},-\phi_{n}\right)\right) & a_{n 3}\left(\mathbf{I}-R\left(\mathbf{n}_{n},-\phi_{n}\right)\right) & \ldots & \ldots \\
a_{32} \sin \frac{\phi_{3}}{2}\left(\cos \frac{\phi_{3}}{2}\left[\mathbf{n}_{3}\right]_{\times}-\sin \frac{\phi_{3}}{2}\left(\left[\mathbf{n}_{3}\right]_{\times}\right)^{2}\right) & -N_{3} \sin \frac{\phi_{3}}{2}\left(\cos \frac{\phi_{3}}{2}\left[\mathbf{n}_{3}\right]_{\times}-\sin \frac{\phi_{3}}{2}\left(\left[\mathbf{n}_{3}\right]_{\times}\right)^{2}\right) & \ldots & \vdots \\
\vdots & & \ldots & \ddots \\
a_{n 2} \sin \frac{\phi_{n}}{2}\left(\cos \frac{\phi_{n}}{2}\left[\mathbf{n}_{n}\right]_{\times}-\sin \frac{\phi_{n}}{2}\left(\left[\mathbf{n}_{n}\right]_{\times}\right)^{2}\right) & a_{n 3} \sin \frac{\phi_{n}}{2}\left(\cos \frac{\phi_{n}}{2}\left[\mathbf{n}_{n}\right]_{\times}-\sin \frac{\phi_{n}}{2}\left(\left[\mathbf{n}_{n}\right]_{\times}\right)^{2}\right) & \ldots & \Omega_{1, n n}
\end{array}\right]
\end{gathered}
$$

Therefore, $\lim _{t \rightarrow \infty} \dot{\mathbf{A}}_{1}(t)=\left[\begin{array}{llll}-a_{12} & -a_{13} & \ldots & -a_{1 n}\end{array}\right]$ $\times P^{-1}(W-P) \mathbf{D}=\left[\begin{array}{lll}-\mathbf{I} & \ldots & -\mathbf{I}\end{array}\right] P^{-1} \Omega \mathbf{D}$. This shows that

$$
\lim _{t \rightarrow \infty}\left\|\dot{\mathbf{A}}_{1}(t)\right\| \leq c_{2}\|\mathbf{D}\| \max _{j=2,3, \ldots, n}\left|\phi_{j}\right|
$$

where $c_{2}$ is a positive constant.

Therefore, the absolute velocities obey $\lim _{t \rightarrow \infty}\left\|\dot{\mathbf{A}}_{1}(t)\right\|=\cdots=\lim _{t \rightarrow \infty}\left\|\dot{\mathbf{A}}_{n}(t)\right\|=$ $O\left(\max _{j=2,3, \ldots, n}\left|\phi_{j}\right|\right)$, as $\phi_{2}, \phi_{3}, \ldots, \phi_{n} \rightarrow 0$. In addition, it is trivial to prove the claim for the case of $\mathbf{D}=0$.

Based on (IV), (V) is obvious.

Remark 5: Theorem 5 shows that the formation distortion and steady velocity of the formation are only related to the maximum of all the mismatch angles, and this fact holds for arbitrarily finite network size.

We next describe simulations which illustrate the validity of Theorem 5. We consider the case three agents with a star graph. In particular, $\mathbf{D}_{12}=[0,-8,0]^{\mathrm{T}}, \mathbf{D}_{23}=[20,0,0]^{\mathrm{T}}$, $\phi_{2}=0.1 \pi$ and $\phi_{3}=0.2 \pi, \mathbf{n}_{2}=\mathbf{n}_{3}=[1,0,0]^{\mathrm{T}}$ and initial states of all the agents are chosen randomly. Figures 5 and 6 show the positions and velocities of the agents during the time interval $[0,100]$. The relative velocities converge to zero, indicating that the agents converge to a fixed formation. However, due to the existence of mismatched coordinate frame, the agents keep moving with non-zero constant absolute velocities and the final formation is distorted from the desired one.

\section{Estimation and compensation algorithms in the n-agent case}

Compensation is slightly more complicated for a general graph. The TRIAD algorithm again gives the rotation matrix linking the coordinate bases of two neighbor agents. By using a tree contained in the undirected graph $\mathcal{G}$, a unique path can be found from node 1 to node $j$, and the rotation matrix linking the coordinate bases of 1 and $j$ is given by the product of the rotation matrices associated with each edge along the path. In this way, the necessary compensation matrices are straightforwardly determined and the necessary compensation introduced.

\section{CONCLUDING REMARKS}

This paper studied the formation shape control problem with mismatched orientations in three-dimensional space.

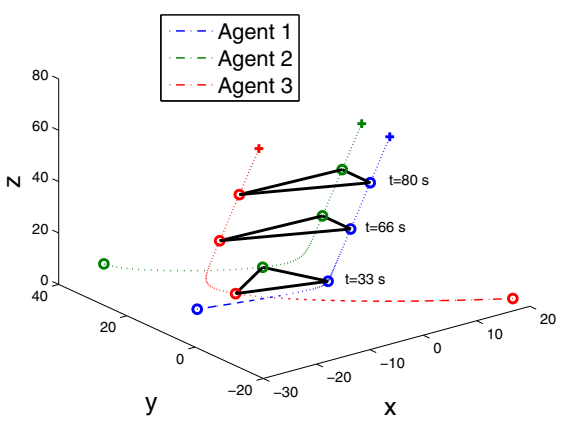

(a) The dotted lines denote the trajectories of the positions of the agents. The circles and the solid black lines denote respectively, the positions of the agents and the formation shape at $\mathrm{t}=33 \mathrm{~s}, \mathrm{t}=66 \mathrm{~s}$, and $\mathrm{t}=80 \mathrm{~s}$.

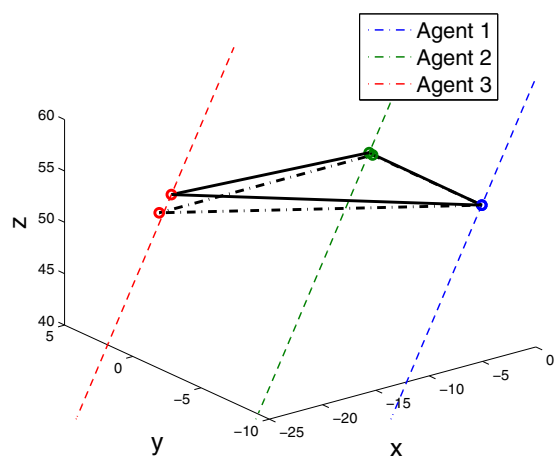

(b) The solid black line and the dotted black line denote respectively, the actual formation shape and desired formation shape at $\mathrm{t}=80 \mathrm{~s}$.

Fig. 5. Three agent case with $\mathbf{D} \neq 0$.

Such a mismatch is a consequence of the fact that it is not physically realistic to claim that all agents have a common coordinate axis orientations when obtaining relative position measurement. We examined the consequences of the mismatched orientations on a standard formation shape control algorithm. The two agent case was first studied and we showed that the agents converge to a fixed, but distorted formation exponentially fast. Unlike the matched case, the formation is not asymptotically stationary. The shape error between the actual final formation and the desired formation was established for small mismatched orientation. We then proposed estimation and compensation algorithms such that 

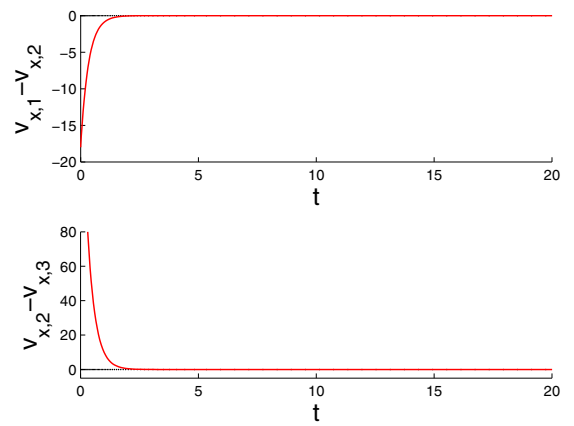

(a) The convergence of relative velocities of the agents.
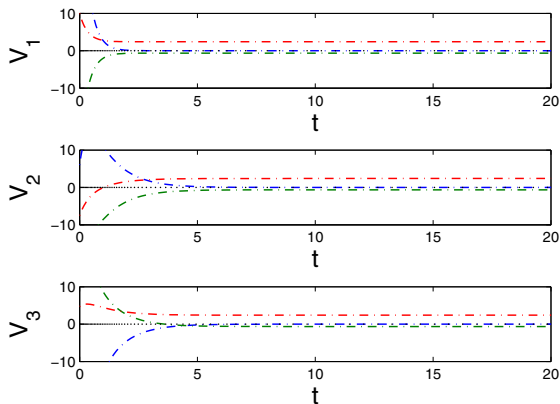

(b) The convergence of absolute velocities of the agents.

Fig. 6. Three agent case with $\mathbf{D} \neq 0$.

the desired formation shape is achieved using the TRIAD algorithm. Furthermore, the extensions to the $n$-agent case and second-order dynamics were investigated. Simulations are provided to validate the theoretical results. Future works include giving more noise-robust algorithms for estimation and compensation, discussing the case of relative velocity feedback for second-order dynamics, studying the situation of a rotating body frame, and solving the leader-following formation control problem with mismatched measurements.

\section{REFERENCES}

[1] D. V. Dimarogonas and K. J. Kyriakopoulos, "On the rendezvous problem for multiple nonholonomic agents," IEEE Transactions on Automatic Control, vol. 52, no. 5, pp. 916-922, 2007.

[2] D. Sieber, F. Deroo, and S. Hirche, "Formation-based approach for multi-robot cooperative manipulation based on optimal control design," in IEEE/RSJ International Conference on Intelligent Robots and Systems (IROS), Tokyo, Japan, 2013, pp. 5227-5233.

[3] W. Ren, "Multi-vehicle consensus with a time-varying reference state," Systems \& Control Letters, vol. 56, no. 7, pp. 474-483, 2007.

[4] G. Shi and Y. Hong, "Global target aggregation and state agreement of nonlinear multi-agent systems with switching topologies," Automatica, vol. 45, no. 5, pp. 1165-1175, 2009.

[5] Y. Hatano and M. Mesbahi, "Agreement over random networks," IEEE Transations on Automatic Control, vol. 50, no. 11, pp. 1867-1872, 2005.

[6] L. Moreau, "Stability of multi-agent systems with time-dependent communication links," IEEE Transactions on Automatic Control, vol. 50, no. 2, pp. 169-182, 2005.

[7] Z. Lin, M. Broucke, and B. Francis, "Local control strategies for groups of mobile autonomous agents," IEEE Transactions on Automatic Control, vol. 49, no. 4, pp. 622-629, 2004.
[8] B. Jiang, M. Deghat, and B. D. O. Anderson, "Translational velocity consensus using distance-only measurements," in Proceedings of 2013th IEEE Conference on Decision and Control, Florence, Italy, 2013, pp. 2746-2751.

[9] R. Olfati-Saber, J. A. Fax, and R. M. Murray, "Consensus and cooperation in networked multi-agent systems," Proceedings of the IEEE, vol. 95, no. 1, pp. 215-233, 2007.

[10] W. Ren, R. W. Beard, and E. M. Atkins, "Information consensus in multivehicle cooperative control: collective group behavior through local interaction," IEEE Control Systems Magazine, vol. 27, no. 2, pp. 71-82, 2007.

[11] L. Krick, M. E. Broucke, and B. A. Francis, "Stabilization of infinitesimally rigid formations of multi-robot networks," International Journal of Control, vol. 82, no. 3, pp. 49-95, 2009.

[12] M. Cao, A. S. Morse, C. Yu, B. D. O. Anderson, and S. Dasgupta, "Maintaining a directed, triangular formation of mobile autonomous agents," Communications in Information and Systems, vol. 11, no. 1, pp. 1-16, 2011.

[13] M. A. Belabbas, S. Mou, A. S. Morse, and B. D. O. Anderson, "Robustness issues with undirected formations," in Proceedings of the 2012th IEEE Conference on Decision and Control, Maui, Hawaii, USA, 2012, pp. 1445-1450.

[14] Z. Sun, S. Mou, B. D. O. Anderson, and A. S. Morse, "Non robustness of gradient control for 3-d undirected formations with distance mismatch," in Proceedings of 2013th Australian Control Conference, Perth, Australia, 2013, pp. 369-374.

[15] Z. Meng, B. D. O. Anderson, and S. Hirche, "Formation control with mismatched compasses," Automatica, vol. 69, pp. 232-241, 2016.

[16] — "Analysis of undirected formation shape control with directional mismatch," in 54th IEEE Conference on Decision and Control, Osaka, Japan, 2015, pp. 6773-6778.

[17] K.-K. Oh and H.-S. Ahn, "Formation control and network localization via orientation alignment," IEEE Transactions on Automatic Control, vol. 59, no. 2, pp. 540-545, 2014.

[18] M. D. Shuster, "A survey of attitude representations," The Journal of the Astronautical Sciences, vol. 41, no. 4, pp. 439-517, 1993.

[19] N. Chaturvedi, A. Sanyal, and N. McClamroch, "Rigid-body attitude controls," IEEE Control Systems Magazine, vol. 31, no. 3, pp. 30-51, 2011.

[20] J. A. Fax and R. M. Murray, "Information flow and cooperative control of vehicle formations," IEEE Transactions on Automatic Control, vol. 49, no. 9, pp. 1465-1476, 2004.

[21] Y. Cao, D. Stuart, W. Ren, and Z. Meng, "Distributed containment control for multiple autonomous vehicles with double-integrator dynamics: Algorithms and experiments," IEEE Transactions on Control Systems Technology, vol. 19, no. 4, pp. 929-938, 2011.

[22] H. K. Khalil, Nonlinear Systems. Prentice Hall, 2002.

[23] H. D. Black, "A passive system for determining the attitude of a satellite," AIAA Journal, vol. 2, pp. 1350-1351, 1964.

[24] M. D. Shuster and S. D. Oh, "Three-axis attitude determination from vector observations," Journal of Guidance, Control, and Dynamics, vol. 4, no. 1, pp. 70-77, 1981.

[25] F. L. Markley, "Attitude determination using vector observations and the singular value decomposition," Journal of the Astronautical Sciences, vol. 36, no. 3, pp. 245-258, 1988.

[26] — - "Attitude determination using vector observations: A fast optimal matrix algorithm," Journal of the Astronautical Sciences, vol. 41, no. 2, pp. 261-280, 1993.

[27] D. Mortari, "Euler-q algorithm for attitude determination from vector observations," Journal of Guidance, Control, and Dynamics, vol. 21, no. 2, pp. 328-334, 1998.

[28] K. Peng and Y. Yang, "Leader-following consensus problem with a varying-velocity leader and time-varying delays," Physica A, vol. 388, no. 2-3, pp. 193-208, 2009. 